\title{
Pan-ethnic carrier screening and prenatal diagnosis for spinal muscular atrophy: clinical laboratory analysis of $>72400$ specimens
}

\author{
Elaine A Sugarman ${ }^{1}$, Narasimhan Nagan ${ }^{\star 1}$, Hui Zhu ${ }^{1}$, Viatcheslav R Akmaev ${ }^{1}$, Zhaoqing Zhou ${ }^{1}$, \\ Elizabeth M Rohlfs ${ }^{1}$, Kerry Flynn ${ }^{1}$, Brant C Hendrickson ${ }^{1}$, Thomas Scholl ${ }^{1}$, Deborah Alexa Sirko-Osadsa ${ }^{1}$ \\ and Bernice A Allitto ${ }^{1}$
}

\begin{abstract}
Spinal muscular atrophy (SMA) is a leading inherited cause of infant death with a reported incidence of $\sim 1$ in 10000 live births and is second to cystic fibrosis as a common, life-shortening autosomal recessive disorder. The American College of Medical Genetics has recommended population carrier screening for SMA, regardless of race or ethnicity, to facilitate informed reproductive options, although other organizations have cited the need for additional large-scale studies before widespread implementation. We report our data from carrier testing $(n=72453)$ and prenatal diagnosis $(n=121)$ for this condition. Our analysis of large-scale population carrier screening data $(n=68471)$ demonstrates the technical feasibility of high throughput testing and provides mutation carrier and allele frequencies at a level of accuracy afforded by large data sets. In our United States pan-ethnic population, the calculated a priori carrier frequency of SMA is 1/54 with a detection rate of $91.2 \%$, and the pan-ethnic disease incidence is calculated to be $1 / 11000$. Carrier frequency and detection rates provided for six major ethnic groups in the United States range from $1 / 47$ and $94.8 \%$ in the Caucasian population to $1 / 72$ and $70.5 \%$ in the African American population, respectively. This collective experience can be utilized to facilitate accurate pre- and post-test counseling in the settings of carrier screening and prenatal diagnosis for SMA.
\end{abstract}

European Journal of Human Genetics (2012) 20, 27-32; doi:10.1038/ejhg.2011.134; published online 3 August 2011

Keywords: spinal muscular atrophy (SMA); pan-ethnic; carrier screening; SMN1

\section{INTRODUCTION}

Spinal muscular atrophy (SMA) is a severe neuromuscular disease characterized by degeneration of the anterior motor neurons, leading to progressive muscle weakness and paralysis. SMA is the leading inherited cause of infant death ${ }^{1}$ with an incidence of $\sim 1 / 10000 .^{2}$ Childhood SMA is subdivided based on age of onset and clinical severity into three types. ${ }^{3}$ Approximately $60 \%$ of SMA patients have type I (Werdnig-Hoffman) disease, ${ }^{4}$ with severe generalized muscle weakness and hypotonia presenting at birth or within the first few months of life and respiratory failure leading to death or permanent ventilator support by 2 years of age. Type II accounts for $\sim 27 \%$ of $\mathrm{SMA},{ }^{4}$ and is variable, with some children having severe respiratory insufficiency and transient ability to sit, whereas others have milder respiratory involvement and are mobile with mechanical support. Individuals with type III SMA (Kugelberg-Welander) experience delayed motor milestones, mild muscle weakness and fatigue.

SMA is caused by mutations in the survival motor neuron 1 (SMN1) gene. ${ }^{4}$ The SMN1 gene is located in a complex region of $5 \mathrm{q} 13$ containing SMN2, a homologous pseudogene of SMN1. SMN1 and SMN2 differ by five nucleotides, one of which is in the coding region, in exon 7 . This sequence change affects splicing resulting in reduced expression of full-length functional protein from the SMN2 gene. $^{5}$ The homozygous absence of $S M N 1$, due to deletion or gene conversion (of SMN1 to SMN2) is responsible for $\sim 95 \%$ of SMA.
Most of the remaining patients are compound heterozygotes with a deletion/gene conversion of the SMN1 gene paired with an intragenic mutation. Affected individuals lacking functional SMN1 retain at least 1 copy of SMN2. An inverse relationship between disease severity and SMN2 copy number in affected individuals has been observed. ${ }^{6}$ Other modifying factors, including SMN2 sequence variants, may also influence phenotypic variability. ${ }^{7}$

Among normal alleles, 1-copy and 2-copy chromosomes are designated as ' 1 ' $(b)$ and ' 2 ' $(c)$, respectively. ${ }^{4,8}$ Chromosomes resulting from a deletion or gene conversion are referred to as a ' 0 ' $(a)$, whereas those with subtle SMN1 intragenic mutations are referred to as ' 1 d' $(d) .{ }^{4}$

Determination of $S M N 1$ copy number in a general carrier screening population permits identification of the majority of SMA carriers before the birth of an affected child. Initial reports of SMN1 copy number quantification focused on diagnostic testing for affected individuals without a homozygous deletion and carrier testing for individuals with a family history of SMA. ${ }^{9}$ In 2002, Ogino et al ${ }^{2}$ reported testing 663 asymptomatic individuals by SMN1 copy number analysis, the majority $(71 \%)$ of whom had a family history of confirmed or suspected SMA. Subsequently, ${ }^{10-12}$ studies from around the world including Australia, ${ }^{13}$ Korea, ${ }^{14}$ Taiwan, ${ }^{11}$ Israel,,${ }^{15,16}$ China ${ }^{17}$ and the United States ${ }^{18}$ have called for screening individuals without a family history of SMA for carrier status, citing disease severity and a high pan-ethnic carrier frequency. In 2008, the American College of

${ }^{1}$ Genzyme Genetics ${ }^{S M}$, Molecular Diagnostic Laboratory, Esoterix Genetic Laboratories, LLC, successor to Genzyme Genetics ${ }^{\text {SM }}$, Westborough, MA, USA

*Correspondence: Dr N Nagan, Genzyme Genetics SM, Molecular Diagnostics Laboratory, 3400 Computer Drive, Westborough, MA 01581 , USA. Tel: +1 508 3898351 ; Fax: +1 508389 5548; E-mail: Narasimhan.Nagan@genzymegenetics.com

Received 24 March 2011; revised 16 May 2011; accepted 13 June 2011; published online 3 August 2011 
Medical Genetics (ACMG) issued practice guidelines recommending all couples be offered SMA carrier screening, regardless of race or ethnicity, with the goal of allowing identified carriers to make informed reproductive choices. ${ }^{19}$ The guidelines identified SMA as meeting generally accepted criteria for a successful screening program including clinical severity, a high frequency of carriers in the screened population, reliable testing with high sensitivity and specificity, available prenatal diagnosis and access to genetic counseling. In 2009, a conference at the US National Institutes of Health reviewed the scientific basis of SMA carrier screening and concluded that panethnic carrier screening for SMA is technically feasible. ${ }^{20}$ Recently, the Association for Molecular Pathology (AMP) issued a statement recognizing the utility and feasibility of population-based SMA carrier screening. ${ }^{21}$ Among the specific recommendations identified were the needs to offer pilot screening programs and determine SMA carrier frequency among different ethnic groups so as to improve risk assessment and post-test counseling. In 2009, the American College of Obstetricians and Gynecologists (ACOG) recommended restricting carrier screening to individuals with a family history of SMA. ACOG asserted that assessment of pilot programs, educational materials, cost effectiveness of screening and absence of laboratory standards and guidelines should be considered before widespread implementation of a carrier screening program for SMA. ${ }^{22}$ Several recent reports address these issues. ${ }^{18,20}$

We report our SMA carrier screening data for $>68400$ individuals without a family history of SMA. Our findings indicate a rapid test uptake beginning before the ACMG guidelines, and further support patient interest in the availability of SMA carrier screening. Furthermore, our data permit refinement of carrier frequency and detection rate information for six major ethnic groups and the general, pan-ethnic population and address the call for a large-scale population screening study.

\section{MATERIALS AND METHODS}

\section{Patient's samples}

Clinical laboratory data were reviewed for 72453 individuals and 121 fetal samples referred for SMN1 copy number analysis over a 12-month time period beginning in May 2008. Relevant information including the clinical indication for testing, family history and ethnicity was obtained by review of the test requisition forms. All individuals referred for testing were reportedly asymptomatic. For clinical testing, it is standard for the referring physician to obtain informed consent, therefore an ethics approval was not required. Individuals referred for testing spanned 44 US states, the District of Columbia and Puerto Rico.

\section{Quantitative real time PCR analysis and sequencing}

DNA was isolated from blood specimens, using a modification of the Qiagen QIAmp 96 DNA Blood Kit (Qiagen GmbH, Hilden, Germany, http://www. qiagen. com). DNA from prenatal cultured AF or CVS specimens was isolated using a modified salting-out method. Multiplex amplifications and qPCR measurements were performed on the ABI Prism 7900HT Real-Time PCR system (Applied Biosystems, Foster City, CA, USA). Each reaction utilized standard Taqman PCR reagents in total volume of $20 \mu \mathrm{l}$ containing $180 \mathrm{ng}$ of DNA, $0.9 \mu \mathrm{m}$ of SMN1 primers (SMN1FP-5'-ATAGCTATTTTTTTTAACTT CCTTTATTTTCC- $3^{\prime}$ and SMN1RP-5'-CTTACTCCTTAATTTAAGGAATGTG AGCA- $3^{\prime}$ ) and each of two internal standard reference gene primers (SMA RCC1FP-5'-AGGTACCACTGGAATTGGTTGAA-3', SMARCC1RP-5'-CATATA TTAACCCTGTCCCTTAAAAGCA-3', SUPT5HFP-5'-CACGTGAAGGTGATT GCTGG-3', SUPT5HRP-5'-CGACCCTTCTATCCACCTACCTC-3'), $0.2 \mu \mathrm{M}$ each of the SMN1-specific FAM-TAMRA hydrolysis probe (5'-AGGGTTTCAG ACAAAATCAAAAAGAAGGAAG- $3^{\prime}$ ), reference gene-specific VIC-TAMRA hydrolysis probes (SMARCC1-5'-AGTACAAGAAGCAGCACGAGCCTCTG-3' and SUPT5- $5^{\prime}$-CGTTATCCTGTTCTCTGACCTCACCATG- ${ }^{\prime}$ ) and a competitive, non-fluorescent, non-hydrolysable probe specific for SMN2 (5'-AGGGTTTTA GACAAAATCAAAAAGAAGGAAGG- $3^{\prime}$ ). Thermal cycling conditions included an initial denaturation of $10 \mathrm{~min}$ at $95^{\circ} \mathrm{C}$ followed by 40 cycles of $15 \mathrm{~s}$ at $95^{\circ} \mathrm{C}$ and $1 \mathrm{~min}$ at $60^{\circ} \mathrm{C}$. Sequencing of primer and probe binding sites was performed on all prenatal specimens, and blood specimens with an SMN1 copy number of $<2$, by bidirectional sequence analysis using BigDye Terminator Cycle Sequencing Kit (version 3.1) followed by capillary electrophoresis (Applied Biosystems, Carlsbad, CA, USA).

Data evaluation was performed using the $\mathrm{C}_{\mathrm{q}}$ (quantification cycle) data exported from SDS 2.2 software (Applied Biosystems, Foster City, CA,

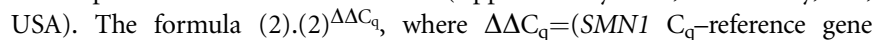
$\mathrm{C}_{\mathrm{q}}$ )-average $\Delta \mathrm{C}_{\mathrm{q}}$ of 2-copy calibrators, was utilized to estimate the SMN1 copy number. Each control and test sample included a copy number CV (coefficient of variation) cutoff of 0.15 between replicate measurements. All results could be assigned to validated, non-overlapping genotype groups of 0,1 , 2 or $\geq 3$ copies of SMN1.

\section{Statistical analysis}

The $95 \%$ confidence intervals around genotype frequency estimates (Table 1) were calculated based on the exact beta distribution model. The derived allele frequencies (Table 2) are maximum likelihood estimates calculated from observed genotype data under assumption of Hardy-Weinberg equilibrium. An EM algorithm was employed to account for missing observations of 0-copy SMN1 genotype in the screening population. The algorithm converged to six significant digits in estimating allele frequencies after two iterations. The $95 \%$ confidence interval around allele frequency estimates and the previous risk estimates (Tables 2 and 3) were calculated as the corresponding percentiles of simulated populations of allele frequencies and risk estimates. These Monte Carlo simulations were based on 10000 random genotype observations generated from the posterior beta distribution followed by maximum

Table 1 Observed SMN1 genotype frequencies among carrier screening referrals $(n=68471)$

\begin{tabular}{|c|c|c|c|c|c|c|c|c|c|c|}
\hline \multirow[b]{2}{*}{ Ethnicity } & \multicolumn{3}{|c|}{ 1-copy } & \multicolumn{3}{|c|}{ 2-copy } & \multicolumn{3}{|c|}{$\geq 3$-copy } & \multirow[b]{2}{*}{ Total } \\
\hline & $n$ & $\%$ & $95 \% \mathrm{Cl}$ & $n$ & $\%$ & $95 \% \mathrm{Cl}$ & $\mathrm{n}$ & $\%$ & $95 \% \mathrm{Cl}$ & \\
\hline Pan-ethnic ${ }^{a}$ & 1162 & 1.7 & $(1.6-1.8)$ & 58094 & 84.84 & $(84.6-85.1)$ & 9215 & 13.46 & $(13.2-13.7)$ & 68471 \\
\hline Caucasian & 494 & 2.02 & $(1.9-2.2)$ & 22252 & 90.93 & $(90.6-91.3)$ & 1725 & 7.05 & $(6.7-7.4)$ & 24471 \\
\hline Ashkenazi Jewish & 78 & 1.34 & $(1.1-1.7)$ & 4913 & 84.62 & $(83.7-85.5)$ & 815 & 14.04 & $(13.2-15.0)$ & 5806 \\
\hline Asian & 73 & 1.57 & $(1.3-2.0)$ & 4148 & 89.26 & $(88.4-90.1)$ & 426 & 9.17 & $(8.4-10.0)$ & 4647 \\
\hline Hispanic & 101 & 1.32 & $(1.1-1.6)$ & 6406 & 83.68 & $(82.9-84.5)$ & 1148 & 15.0 & $(14.2-15.8)$ & 7655 \\
\hline Asian Indian & 17 & 1.74 & $(1.1-2.8)$ & 808 & 82.79 & $(80.4-85.1)$ & 151 & 15.47 & $(13.4-17.9)$ & 976 \\
\hline African American & 48 & 0.98 & $(0.7-1.3)$ & 2536 & 51.94 & $(50.5-53.3)$ & 2299 & 47.08 & $(45.7-48.5)$ & 4883 \\
\hline Not provided & 290 & 1.68 & $(1.5-1.9)$ & 14645 & 84.97 & $(84.4-85.5)$ & 2300 & 13.35 & $(12.8-13.9)$ & 17235 \\
\hline
\end{tabular}

Abbreviation: $\mathrm{Cl}$, confidence interval.

a2798 individuals of mixed or other ethnicity are included in the pan-ethnic total of 68471 . 
Table 2 Derived SMN1 allele frequencies

\begin{tabular}{|c|c|c|c|c|c|c|c|}
\hline Ethnicity (number of individuals) & O-copy (\%) (a) & $95 \% \mathrm{Cl}(\%)$ & $1-\operatorname{copy}(\%)(b)$ & $95 \% \mathrm{Cl}(\%)$ & $2-\operatorname{copy}(\%)(c)$ & $95 \% \mathrm{Cl}(\%)$ & $1^{d}(\%)(d)$ \\
\hline Pan-ethnic $(N=68471)$ & 0.92 & $(0.87-0.98)$ & 92.02 & (91.86-92.17) & 7.04 & $(6.90-7.18)$ & 0.02 \\
\hline Caucasian $(N=24471)$ & 1.06 & $(0.97-1.16)$ & 95.3 & (95.09-95.48) & 3.63 & $(3.47-3.80)$ & 0.02 \\
\hline Ashkenazi Jewish $(N=5806)$ & 0.73 & $(0.59-0.91)$ & 91.91 & (91.36-92.39) & 7.34 & $(6.88-7.84)$ & 0.02 \\
\hline Asian $(N=4647)$ & 0.83 & $(0.66-1.05)$ & 94.42 & (93.90-94.87) & 4.74 & $(4.31-5.19)$ & 0.02 \\
\hline Hispanic $(N=7655)$ & 0.72 & $(0.59-0.88)$ & 91.4 & $(90.92-91.86)$ & 7.86 & $(7.43-8.31)$ & 0.02 \\
\hline Asian Indian $(N=976)$ & 0.96 & $(0.60-1.54)$ & 90.88 & (89.36-92.09) & 8.15 & $(6.98-9.48)$ & 0.02 \\
\hline African American ( $N=4883$ ) & 0.68 & $(0.52-0.91)$ & 71.79 & $(70.73-72.76)$ & 27.51 & $(26.57-28.51)$ & 0.02 \\
\hline Not provided $(N=17235)$ & 0.91 & $(0.81-1.03)$ & 92.09 & (91.78-92.38) & 6.98 & $(6.71-7.26)$ & 0.02 \\
\hline
\end{tabular}

Abbreviation: $\mathrm{Cl}$, confidence interval.

a Number of individuals with no family history of SMA referred for SMN1 copy number analysis by ethnic background.

Table 3 Carrier frequency and risk reduction by ethnicity

\begin{tabular}{|c|c|c|c|c|}
\hline Ethnicity & $\begin{array}{c}\text { Detection } \\
\text { rate (\%) }\end{array}$ & $\begin{array}{c}\text { a priori risk } \\
\text { (95\% Cl) }\end{array}$ & $\begin{array}{c}\text { Reduced } \\
\text { risk for } \\
\text { 2-copy result }\end{array}$ & $\begin{array}{c}\text { Reduced } \\
\text { risk for } \\
\geq 3 \text {-copy result }\end{array}$ \\
\hline Pan-ethnic & 91.2 & $1: 54(1: 51-1: 57)$ & $1: 527$ & $1: 5400$ \\
\hline Caucasian & 94.8 & $1: 47(1: 43-1: 51)$ & $1: 834$ & $1: 5600$ \\
\hline Ashkenazi Jewish & 90.5 & $1: 67(1: 54-1: 83)$ & $1: 611$ & $1: 5400$ \\
\hline Asian & 93.3 & $1: 59(1: 47-1: 74)$ & $1: 806$ & $1: 5600$ \\
\hline Hispanic & 90.0 & $1: 68(1: 57-1: 83)$ & $1: 579$ & $1: 5400$ \\
\hline Asian Indian & 90.2 & $1: 52(1: 33-1: 82)$ & $1: 443$ & $1: 5400$ \\
\hline African American & 70.5 & $1: 72(1: 54-1: 94)$ & $1: 130$ & $1: 4200$ \\
\hline Not provided & 91.3 & $1: 54(1: 48-1: 161)$ & $1: 536$ & $1: 5450$ \\
\hline
\end{tabular}

a priori risk includes the $[1+0],\left[1+1^{d}\right],[2+0]$ and $\left[2+1^{d}\right]$ allele pairings for individuals with no family history of spinal muscular atrophy.

likelihood estimation of the allele frequencies under Hardy-Weinberg assumption. The $\chi^{2}$-test with Yates correction was used for comparison of 1-copy genotype frequencies between populations.

\section{RESULTS}

\section{Population-based carrier screening}

Approximately 95\% of the 72453 individuals referred for carrier testing had no family history of SMA. This ethnically diverse carrier screening population was comprised of 68471 individuals, $94 \%$ of whom were female. For analysis purposes, our study population was categorized by ethnicity according to information provided on the test requisition. Ethnicity-specific risk calculations were generated for six ethnic groups (Caucasian, Ashkenazi Jewish, Hispanic, African American, Asian, Asian Indian) and for individuals for whom ethnicity was 'Not Provided' resulting in a total of seven categories. Pan-ethnic calculations included individuals from these categories as well as those reporting multiple or other ethnicities $(n=2798)$. The SMN1 allele frequencies $(a, b, c)$ derived from the observed genotype frequencies (Table 1) are listed in Table 2. For the purpose of this study, the allele frequency of intragenic SMN1 point mutations $(d)$, was presumed to be constant across ethnic groups based upon an earlier report describing SMN1 deletion/gene conversion and point mutation distribution among 501 affected patients. ${ }^{23}$ The calculated SMA carrier frequency and SMN1 deletion detection rate for the pan-ethnic population and each of seven categories are listed in Table 3. The calculated carrier frequency is the a priori risk for an individual to be a carrier and includes the probability of the most likely allele pairings expected to occur among carriers in the general population, namely $[1+0],\left[1+1^{\mathrm{d}}\right],[2+0]$ and $\left[2+1^{\mathrm{d}}\right]$. The SMN1 deletion detection rate (or sensitivity of the carrier test result) is the percentage of carriers with the $[1+0]$ genotype (who will be identified by a 1-copy result), among all carriers of SMA (as determined by the carrier frequency). The reduced carrier risk following a 2- or 3-copy result in an individual with no family history of SMA is listed in Table 3.

The calculated pan-ethnic carrier frequency of $1 / 54$ (Table 3 ) is consistent with the frequency of $1 / 40-1 / 60$ frequently cited in the literature. ${ }^{13}$ The carrier frequencies among each of the ethnic groups ranged from $1 / 47$ in the Caucasian population to $1 / 72$ in the AfricanAmerican population. The carrier detection rate in the pan-ethnic screening population is $91.2 \%$. Among the seven categories for whom prior carrier risk and carrier detection rate is calculated (Table 2), all but African Americans have a detection rate exceeding 90\% and an $a$ priori risk in the range of $1 / 47-1 / 68$.

The carrier detection rate in the African American population is $70.5 \%$. This finding from our large scale clinical experience confirms our earlier observation ${ }^{24}$ of a decreased carrier detection rate (Table 3 ) due to an associated increased 2-copy $(c)$ allele frequency (Table 1) in the African American population. Furthermore, the estimated negative predictive value of the carrier test as calculated by the derived allele pairings, was $>99 \%$ for both the pan-ethnic group as well as each of the six major ethnic subgroups.

\section{Other carrier test indications}

Among 72453 individuals referred for carrier testing, greater than 95\% $(n=68945)$, had an indication for testing provided by the referring physician. The $4.8 \%(n=3508)$ of referrals for whom no indication was provided, were excluded from further analysis, although they were most likely referred for carrier screening, as $95 \%$ of these samples were from women of reproductive age. Fewer than $1 \%(n=329)$ of individuals referred for carrier testing had indications of a family history of SMA (including obligate carriers) or abnormal fetal ultrasound findings. Of the 71 individuals referred for an indication of abnormal fetal ultrasound findings, the most frequent findings were increased nuchal translucency $(n=17)$ and cystic hygroma $(n=4)$ in the first trimester and joint contractures $(n=18)$ and increased nuchal fold $(n=10)$ in the second trimester. Three individuals were identified as carriers; all others, including the partners of these carriers, had a 2- or 3-copy result. Among the three carriers, only one had fetal SMA testing in our laboratory and the fetus had 2 SMN1 copies. These results indicate that SMA may be included as a differential diagnosis following identification of suggestive abnormal fetal ultrasound findings. 


\section{Prenatal diagnosis}

A total of 121 fetal samples (51 cultured chorionic villi and 70 amniocyte) were received for SMN1 copy number analysis. Of the 54 fetuses at a $25 \%$ risk to be affected with SMA, 47 were based on a previous affected child, four on identification of parental carrier status following carrier screening and three had both parents identified as carriers at other laboratories. Fifteen of the $54(27.8 \%)$ were predicted to be affected with SMA, based on a 0 -copy result from testing. This result is consistent with the expected $25 \%$ frequency given autosomal recessive inheritance $(P=0.26)$.

In all, 59 fetuses were tested after identification of carrier status in one parent, with the other parent having a 2- or 3-copy result determined concurrently or before fetal testing. Of these, 27 fetuses had 1 copy and 32 had 2 copies of SMN1. At least 53 of the 59 had an indication for CVS or amniocentesis that was unrelated to the parental SMA carrier testing (eg, advanced maternal age, abnormal maternal serum screen). The remaining six samples were received as cultured cells from outside laboratories. The indication for the prenatal diagnostic procedure in these cases was not readily available, although the average age of these mothers was 37 years. Seven of the 121 fetal samples were referred based on abnormal ultrasound findings such as joint contractures suggestive of SMA, and all had 2-copy fetal results. Lastly, one sample was tested due to a reported family history of SMA, although neither parent was identified as a 1-copy carrier and the fetus had 2 copies of SMN1.

\section{Sequence variants that interfere with SMN1 copy number determination}

To rule out false positive results due to the presence of sequence variants under the primer and probe-binding sites used in our analysis, follow-up sequencing restricted to these sites was performed on all samples with 1 copy of SMN1 and all prenatal specimens regardless of SMN1 copy number. This led to the identification of a recurrent variant, NT_006713.14:c.865T >A (g.29631T > A; p. C289S), in a heterozygous state among 35 individuals. This variant was most frequent among Hispanic individuals $(n=19)$ but was also identified in Ashkenazi Jewish and Caucasian ( $n=9$ combined) individuals and in individuals for whom ethnicity information was not provided to the laboratory $(n=7)$. Another variant, NT_006713.14:c.867C > T (g.29633C > T; p.C289C) was identified in a heterozygous state in one Hispanic individual. Both these variants resulted in a 1-copy result by our real time PCR methodology and were subsequently identified by sequence analysis to be present in a heterozygous state within the SMN1 gene as evidenced by presence of the nucleotide ' $\mathrm{C}$ ' at the critical position, c.840 that distinguishes SMN1 from SMN2. Neither of these variants was observed in a homozygous state among individuals analyzed. Our practice of restricting follow-up sequence analysis to individuals identified to have 1 copy of SMN1 precludes an accurate estimation of the general population allele frequency of these variants.

\section{DISCUSSION}

SMA affects individuals of all ethnicities. The estimated pan-ethnic disease frequency, $(a+d),{ }^{2}$ derived from our carrier frequency data $(1 / 11000$, Table 2$)$ is consistent with the reported prevalence estimate of 1/10000 reported for clinically typical SMA derived from larger population studies. ${ }^{4}$ Previous reports on SMA carrier frequencies in limited populations have identified a discrepancy between the estimated disease prevalence $(1 / 10000)$ and that extrapolated from observed carrier frequency estimates (1/6000) under HardyWeinberg equilibrium. Although possible explanations for the earlier discrepancies have been proposed, ${ }^{4}$ our present study is the first to report a pan-ethnic carrier frequency that is most consistent with the reported incidence of SMA.

For purposes of genetic counseling within the setting of populationbased carrier screening, pan-ethnic carrier frequency (1/54) and carrier detection rate $(91 \%)$ data are particularly useful when ethnicity is unknown or reflects admixture. When ethnicity is known, however, it may be possible to further refine the carrier frequency or detection rate provided in pre-test education and counseling, as well as provide more accurate estimates of residual risk following identification of a 2- or 3-copy SMN1 result. For instance, a Caucasian individual with a 2 -copy result following carrier screening would have an $\sim 1 / 800$ residual risk to be a carrier, whereas an African American individual with the same result would have a 1/130 risk to be a carrier.

Testing 4883 African American individuals with no family history of SMA confirmed earlier observations ${ }^{24}$ of a higher frequency of the 2-copy allele $(c)$ in this population as compared with other populations. This suggests a higher frequency of $[2+0]$ carriers who would not be identified by an assay designed to detect deletion carriers who have 1 copy of SMN1. This is accurately reflected by the lower detection rate of $71 \%$ in this population. The a priori risk for an African American individual to be a carrier $(1 / 72)$ is, however, comparable to other populations and supports the inclusion of African Americans among those being offered carrier screening for SMA, with appropriate counseling regarding the limitations of testing and associated residual risks.

In this study of 7655 Hispanic individuals, the observed SMN1 1 -copy frequency did not differ significantly from that observed in our earlier study ${ }^{24}$ of 1030 individuals $(P=0.1869)$. In the Asian carrierscreening population, pair-wise comparison of observed SMN1 1-copy frequency between our current data set $(n=4647)$ and those of our earlier study ${ }^{24}(n=1027)$, as well as studies in native Chinese ${ }^{17}$ $(n=1712)$ and Korean ${ }^{14}(n=326)$ populations, also did not yield significant differences at $1 \%(P$-values $=0.7776,0.0366,0.5674$, respectively). Among individuals identified as Asian Indian $(n=976)$, the 1-copy genotype frequency was $1.74 \%$ with a calculated a priori carrier frequency of 1/52. We are not aware of previous studies of SMA carrier frequency in the general Asian Indian population. Similarly, the SMN1 1-copy genotype frequency distribution in our Ashkenazi Jewish population was not significantly different from that reported in the Israeli population ${ }^{16}(P=0.6575)$. Finally, an evaluation of genotype frequencies for individuals whose ethnic background was 'Not Provided' on the ordering test requisition supports the conclusion that the ethnicity distribution of this group does not differ from that of the pan-ethnic population as a whole as the 1-copy genotype frequencies are similar $(P=0.9217)$.

At least seven couples with no family history of SMA in our carrierscreening population were identified as carriers with a $25 \%$ risk to have an affected child. As a reference laboratory we do not have complete ascertainment of family samples, and it is possible that some partners of individuals identified as carriers via screening were tested at other laboratories. Additionally, we could have incomplete ascertainment if partners were not identified as such on ordering paperwork or if a fetal sample from carrier parents was tested at another laboratory. Of the seven couples, four had fetal testing in our laboratory and no affected fetuses were identified in this small group.

Among individuals with a family history of SMA, there have been case reports of first-degree relatives with an SMN1 copy number of 0 who are asymptomatic or mildly affected. ${ }^{25}$ This raises the question of the frequency of individuals with 0 copies of SMN1 in the general population. Among the 72453 samples tested, we did not identify any individuals referred for carrier testing with an apparent 0-copy SMN1 result. 
The identification of 36 individuals with sequence variants in the primer/probe region underscores the importance of this additional quality assurance measure to identify potential false positive results. These individuals could be misclassified as being carriers of a deletion/ gene conversion within the SMN1 gene. At present, there is insufficient evidence to classify these variants as either disease causing or benign. Therefore, although the SMN1 copy number of individuals identified with these variants can be reclassified, their SMA carrier status and associated residual risk cannot be accurately determined at present. Follow-up options, such as carrier testing of the partner can be explored. In the absence of follow-up sequencing, the estimated pan-ethnic-positive predictive value of our carrier analysis (TP (1162)/ TP (1162)+FP (36)) would have been $97 \%$.

In our prenatal testing cohort, as expected, $\sim 25 \%$ of the at-risk fetuses were found to have 0 copies of SMN1 and were predicted to be affected with SMA. Among 54 fetal samples referred for testing due to a $25 \%$ risk to be affected, 47 were from obligate carrier parents with a previous affected child. Of these, eight obligate carriers $(8.5 \%)$ had 2 copies of $S M N 1$ and a partner with 1 copy of $S M N 1$. This frequency is consistent with the frequency of $7 / 117(6.0 \%)$ obligate carrier parents identified to have 2 copies of SMN1 as reported by Smith et $a^{18}(P=0.79)$. In our study, additional studies to distinguish the $[2+0]$ versus $[1+1]$ status of these 2 -copy obligate carrier parents were not performed. Therefore, the exact frequency of 2-copy chromosomes in our obligate carrier parent cohort cannot be determined.

A lack of agreement exists among the limited number of studies investigating a relationship between abnormal ultrasound findings and SMA. Some reports suggest an association between increased nuchal translucency and SMA, ${ }^{26}$ although this association has not been supported by all studies. ${ }^{27}$ The inclusion of abnormal ultrasound findings among indications for carrier testing and prenatal diagnosis within our study suggests possible physician interest in including SMA among the differential diagnoses for select ultrasound abnormalities, although we are unable to determine the frequency with which parents or fetuses with these findings are referred for SMA testing.

The most frequent indication for fetal testing (49\%) was having one carrier parent identified during screening. In these circumstances, the other parent was identified as having 2 or 3 copies of SMN1 or was tested for carrier status concurrently with the fetal SMN1 copy number analysis. In all but six samples for which clinical indication could not be confirmed, invasive prenatal diagnosis was performed for a reason unrelated to the carrier parent's SMN1 status. It is presumed that fetal testing was pursued in the interest of time in these cases, as was the case for individuals in whom the fetal sample and the untested parent were analyzed at the same time, or for additional reassurance.

The a priori risk for a fetus to be affected with SMA, when one parent is identified as a 1-copy carrier and the other parent has an SMN1 copy number of 2, can vary by ethnic background. In a Caucasian couple, the risk to have an affected fetus is $1 / 2528$. In contrast, the risk for an African American couple with the same parental results is $1 / 264$. SMN1 copy number analysis for a fetus in this circumstance could reveal a 0-copy SMN1 result, consistent with a prediction for the fetus to be affected. In addition, such a result would set the parental phase for the 2-copy parent as a [2+0] carrier. Alternatively, a 1-copy fetal result in this situation would be associated with an $\sim 1 / 4000$ risk for the fetus to be affected with SMA, due to compound heterozygosity for a $1^{\mathrm{d}}(d)$ allele.

In a pilot study of general population carrier screening in the United States, Prior et al ${ }^{18}$ reported $\sim 60 \%$ of individuals seeking prenatal genetic counseling accepted carrier testing for SMA. After result disclosure, $98.7 \%$ of patients were glad they pursued testing. In Israel, among women electing carrier screening for cystic fibrosis and fragile $\mathrm{X}$ syndrome, a large-scale population screening study found $93 \%$ requested SMA testing as well. ${ }^{15}$ Our clinical laboratory analysis of $>68000$ individuals without a family history of SMA, starting before the 2008 ACMG guideline, demonstrates (1) rapid test uptake by physicians and further supports patient interest in the availability of carrier screening for this disorder; (2) the feasibility of high throughput carrier testing for SMA; and (3) new and valuable information regarding SMN1 copy number in the general United States population to permit more accurate residual carrier risk calculations based on ethnicity-specific carrier frequencies and detection rates.

Furthermore, these data address specific recommendations set forth by professional organizations such as the AMP and ACOG and fully support the ACMG recommendations to offer SMA carrier screening to all, regardless of race or ethnicity.

\section{CONFLICT OF INTEREST}

At the time this study was conducted, all authors were employed by the Genzyme Genetics unit of Genzyme Corporation, and held stock of and/or options with Genzyme Corp. Currently all authors, with the exception of Viatcheslav Akmaev, are employed by Esoterix Genetics Laboratories, LLC, a wholly owned subsidiary of Laboratory Corporation of America Holdings, and may hold stock of and/or stock options with LabCorp.

\section{ACKNOWLEDGEMENTS}

We are thankful to Denise Boldy, Tiffany Colacchio, and the technical and clinical staff of the Molecular Diagnostic Laboratory at Genzyme Genetics.

Genzyme Genetics and its logo are trademarks of Genzyme Corporation and used by Esoterix Genetic Laboratories, LLC, a wholly owned subsidiary of LabCorp, under license. Esoterix Genetic Laboratories and LabCorp are operated independently from Genzyme Corporation.

1 Miniño AM, Xu JQ, Kochanek KD: Deaths: preliminary data for 2008. Natl Vital Stat Rep 2010; 59. National Center for Health Statistics: Hyattsville, MD.

2 Ogino S, Leonard DG, Rennert H, Wilson RB: Spinal muscular atrophy genetic testing experience at an academic medical center. J Mol Diagn 2002; 4: 53-58.

3 Zerres K, Rudnik-Schoneborn S: Natural history in proximal spinal muscular atrophy; clinical analysis of 445 patients and suggestions for a modification of existing classifications. Arch Neurol 1995; 52: 518-523.

4 Ogino S, Wilson RB, Gold B: New insights on the evolution of the SMN1 and SMN2 region: simulation and meta-analysis for allele and haplotype frequency calculations. Eur J Hum Genet 2004; 12: 1015-1023.

5 Lorson CL, Hahnen E, Androphy EJ, Wirth B: A single nucleotide in the SMN gene regulates splicing and is responsible for spinal muscular atrophy. Proc Natl Acad Sci USA 1999; 96: 6307-6311.

6 Feldkötter M, Schwarzer V, Wirth R, Wienker TF, Wirth B: Quantitative analyses of SMN1 and SMN2 based on real-time lightCycler PCR: fast and highly reliable carrier testing and prediction of severity of spinal muscular atrophy. Am J Hum Genet 2002; 70: 358-368.

7 Prior TW, Krainer AR, Hua Y et al: A positive modifier of spinal muscular atrophy in the SMN2 gene. Am J Hum Genet 2009; 85: 408-413.

8 Mailman MD, Hemingway T, Darsey RL et al: Hybrids monosomal for human chromosome 5 reveal the presence of a spinal muscular atrophy (SMA) carrier with two SMN1 copies on one chromosome. Hum Genet 2001; 108: 109-115.

9 McAndrew PE, Parsons DW, Simard LR et al: Identification of spinal muscular atrophy carriers and patients by analysis of $S M N^{T}$ and $S M N^{C}$ gene copy number. Am J Hum Genet 1997; 60: 1411-1422.

10 Anhuf D, Eggermann T, Rudnik-Schoneborn S, Zerres K: Determination of SMN1 and SMN2 copy number using TaqMan technology. Hum Mutat 2003; 22: 74-78.

11 Su YN, Hung CC, Li H et al: Quantitative analysis of SMN1 and SMN2 genes based on DHPLC: a highly efficient and reliable carrier-screening test. Hum Mutat 2005; 25: 460-467.

12 Scarciolla O, Stuppia L, DeAngelis MV et al: Spinal muscular atrophy genotyping by gene dosage using multiple ligation-dependent probe amplification. Neurogenetics 2006; 7: 269-276. 
13 Smith M, Calabro V, Chong B, Gardiner N, Cowie S, du Sart D: Population screening and cascade testing for carriers of SMA. Eur J Hum Genet 2007; 15: 759-766.

14 Yoon S, Lee CH, Lee KA: Determination of SMN1 and SMN2 copy numbers in Korean population using multiplex ligation dependent probe amplification. Korean $\mathrm{J}$ Lab Med 2010; 30: 93-96.

15 Ben-Shachar S, Orr-Urtreger A, Bardugo E et al: Large-scale population screening for spinal muscular atrophy: Clinical implications. Genet Med 2011; 13: $110-114$.

16 Sukenik-Halevy R, Pesso R, Garbian N, Magal N, Shohat M: Large scale population carrier screening for spinal muscular atrophy in Israel-effect of ethnicity on the falsenegative rate. Genet Test Mol Biomarkers 2010; 14: 319-324.

17 Sheng-Yuan Z, Xiong F, Chen Y-J et al: Molecular characterization of SMN copy number derived from carrier screening and from core families with SMA from a Chinese population. Eur J Hum Genet 2010; 18: 978-984.

18 Prior TW, Snyder PJ, Rink BD et al: Newborn and carrier screening for spinal muscular atrophy. Am J Med Genet A 2010; 152A: 1608-1610.

19 Prior TW: Carrier screening for spinal muscular atrophy. Genet Med 2008; 10: 20-26.

20 Gitlin J, Fishbeck K, Crawford TO et al: Carrier testing for spinal muscular atrophy. Genet Med 2010; 10: 621-622.

21 Muralidharan K, Wilson R, Ogino S, Nagan N, Curtis C, Schrijver I: Population carrier screening for spinal muscular atrophy: A position statement of the Association for Molecular Pathology. J Mol Diag 2011; 13: 3-6.
22 ACOG Committee Opinion: Spinal Muscular Atrophy. Obstet Gynecol 2009; 113 : 1194-1196.

23 Wirth B, Herz M, Wetter A et al: Quantitative analysis of survival motor neuron copies: identification of subtle SMN1 mutations in patients with spinal muscular atrophy, genotype-phenotype correlation, and implications for genetic counseling. Am J Hum Genet 1999; 64: 1340-1356.

24 Hedrickson BC, Donohoe C, Akmaev VR et al: Differences in SMN1 allele frequencies among ethnic groups within North America. J Med Genet 2009; 46: 641-644.

25 Prior T, Swoboda KJ, Scott HD, Hejmanowski AQ: Homozygous SMN1 deletions in unaffected family members and modification of the phenotype by SMN2. Am J Med Genet 2004; 130A: 307-310.

26 Bilardo CM, Timmerman E, Pajkrt E, van Maarle M: Increased nuchal translucency in euploid fetuses-what should we be telling parents? Prenat Diagn 2010; 30: 93-102.

27 Zadeh N, Hudgins L, Norton M: Nuchal translucency measurement in fetuses with spinal muscular atrophy. Prenat Diagn 2011; 31: 327-330.

\section{(c)}

This work is licensed under the Creative Commons Attribution-NonCommercial-No Derivative Works 3.0 Unported Licence. To view a copy of this licence, visit http:// creativecommons.org/licenses/by-nc-nd/3.0/ 